\title{
Almost Periodic Hill's Equation and the Rings of Saturn
}

\author{
Joseph E. Avron ${ }^{(a)}$ and Barry Simon ${ }^{(b)}$ \\ Department of Mathematics, California Institute of Technology, Pasadena, California 91125
}

(Received 30 January 1981)

\begin{abstract}
Incommensurate perturbations of classical orbits lead to an almost periodic Hill's operator whose spectrum, we argue, is a Cantor set, but one with large Lebesgue measure. Applied to the rings of Saturn, this implies that the complex groove structure in the rings approximates a Cantor set. We also emphasize the possible relevance of the sun in producing "side gaps" which magnify the apparent gap size.

PACS numbers: $95.10 . \mathrm{Ce}, 03.65 . \mathrm{Db}, 96.30 . \mathrm{Mh}$
\end{abstract}

One of the striking discoveries of Voyager I is that the structure of the rings of Saturn is exceedingly complex. Prior to this information, one of the more popular explanations of the structure of the rings was as an effect of resonances with the moons of Saturn. ${ }^{1}$ Our goal here is to examine whether a resonance picture can possibly provide a structure as complicated as that observed. We will study the question within a linear stability analysis which will lead to the spectral analysis of an almost periodic Hill's equation. Our conclusion is that the structure of such a spectrum is sufficiently rich so that it may account for the main features of the ring structure. More definitive conclusions will have to await, among other things, further study of this spectral analysis which we hope this note will stimulate.

Linear stability is an approximation and as such does not have all the deep structure of the fully nonlinear equations of classical mechanics, as bornout by the Kolmogorov-Arnold-Moser (KAM) theory. ${ }^{2}$ However, it has the advantages of being conceptually simple, relatively easy to estimate orders of magnitude and the right qualitative features. Indeed, one can regard certain aspects of our analysis as a kind of "poor man's KAM." Moreover, since the basic equation turns out to be a time-independent Schrodinger equation (see below), one can use intuition ${ }^{3}$ from that subject in this analysis even though the analogy is on the level of the mathematics and not on the basic physics.

The main features of the theory of the rings of Saturn which emerges are as follows:

(1) The largest individual gaps are on the order of $20 \mathrm{~km}^{4}$ and such large gaps only occur near the position of a classical resonance, e.g., at the Cassini division.

(2) In an idealized model where one considers point particles running for an infinite time, with no collisions, the rings have the structure of a Cantor set (see below), i.e., the gaps are dense.
(3) Large scale features like the Cassini division are aggregates of a large number of gaps separated by thin ringlets, something consistent with the Voyager observations. We will argue that a long period can conspire with a moon resonance to produce resonance "side gaps" and therefore an aggregate of gaps. One candidate ${ }^{5}$ for this long period term is the sun.

Let us recall Hill's basic analysis of linear stability. Consider a closed orbit, $\overrightarrow{\mathrm{v}}(t)$, in the $x-y$ plane of Newton's equation $\ddot{\vec{v}}=\overrightarrow{\mathrm{F}}[\overrightarrow{\mathrm{v}}(t)]$. If we replace $\overrightarrow{\mathrm{v}}(t)$ by $\overrightarrow{\mathrm{v}}(t)+\hat{z} w(t)$ and keep first order terms in $w$ we find $H w=0$ with

$$
H=-\left(d^{2} / d t^{2}\right)+V(t)
$$

where $V(t) \equiv-\partial_{z} F_{z}[\overrightarrow{\mathrm{v}}(t)]$. If $H$ has zero in its (continuous) spectrum $w$ stays bounded; otherwise, there is a solution which grows exponentially. If the original orbit is not in a plane or if $w$ is not perpendicular to that plane, $H$ is replaced by a vector-valued Hill's operator.

To describe our picture of the spectrum of the relevant Hill's operator, we first describe certain Cantor sets.

A Cantor set is a subset of the real line which is closed, has no isolated points, and which is nowhere dense, i.e., whose complement is dense. The original set constructed by Cantor had the additional feature of zero-Lebesgue measure. In contrast we shall concentrate on Cantor sets with large Lebesgue measure. An example is as follows: Pick a sequence of odd (positive) integers $n_{j}=2 l_{j}+1$ and expand $x=l_{0}+\sum_{j=1}^{\infty} a_{j} /\left(n_{1} \cdots n_{j}\right), a_{j}$ $=0, \ldots, n_{j}-1$. The set of points with no $a_{j}=l_{j}$ is a Cantor set and its Lebesgue measure in $\left[l_{0}, l_{0}\right.$ $+1]$ is $\prod_{j=1}^{\infty}\left(1-n_{j}^{-1}\right)$, which will be close to 1 if $\sum n_{j}^{-1}$ is small.

We shall argue that the spectrum of (1) with $V(t)$ quasiperiodic is a Cantor set with a large Lebesgue measure. By quasiperiodic we mean $V(t)=f\left(\omega_{1} t, \ldots, \omega_{n} t\right), f\left(t_{1}+m_{1}, \ldots, t_{n}+m_{n}\right)=f\left(t_{1}\right.$, $\left.\ldots, t_{n}\right), m_{i}$ integers and $\omega_{i}$ incommensurate. The 
evidence we have for this claim is the following ${ }^{6}$ :

(1) For weak coupling, i.e., $V(t)=g f(t), g$ small, take $f(t)=\sum \tilde{f}(\overrightarrow{\mathrm{m}}) e^{2 \pi i \mathrm{~m} \cdot \vec{t}}$ with $\tilde{f}(\overrightarrow{\mathrm{m}}) \neq 0,|\overrightarrow{\mathrm{m}}|$ $\geqslant m_{0}$, and $\sum|\tilde{f}(\overrightarrow{\mathrm{m}})|<\infty$. First order perturbation theory ${ }^{7}$ gives gaps of order $g|\tilde{f}(\overrightarrow{\mathrm{m}})| \neq 0$ centered at energies $E_{\overrightarrow{\mathrm{m}}}=(\pi \overrightarrow{\mathrm{m}} \cdot \vec{\omega})^{2}$ whose total measure is small. The $E_{\overrightarrow{\mathrm{m}}}$ are dense in $[0, \infty)$.

(2) There is a different class of almost periodic potentials (limit-periodic ${ }^{8}$ ) for which Cantor spectrum with large Lebesgue measure has been proven rigorously by Moser $^{9}$ and independently by Avron and Simon. ${ }^{10}$

(3) Azbel $^{11}$ analyzed yet another class of potentials in the strong coupling limit. He argues that the spectrum has all the scales of the continued fraction expansion of the periods, something compatible with a Cantor set.

(4) A lattice version of Eq. (1) (a difference equation) has been analyzed numerically by Hof stadter ${ }^{12}$ in another context and suggests a Cantor set for the spectrum.

Since Cantor sets have no scale, what appears to be a wide band on one scale is, in fact, several bands separated by narrow gaps on a finer scale. The aggregation of small individual gaps and bands to form apparent gaps and bands on a larger scale plays an important role in the sequel. To see how this may come about consider the spectrum of the Schrodinger operator

$$
\left(-d^{2} / d t^{2}\right)+\lambda \cos (2 \pi t)+\mu \cos (2 \pi t / n),
$$

with $\lambda$ and $\mu$ fairly small, and $n$ a large number so that $\mu n$ is not too small. We take $n$ an integer so we can analyze (2) in terms of Floquet theory although we assume the qualitative features we find are true even if $n$ is nonintegral. Consider the gaps near energy 1 . If $\mu=0$, there is one gap of size $\lambda$. If $\lambda=0$, there are many gaps at energy approximately $[(n-k) / n]^{2}(k$ an integer near 0$)$ but the asymptotics of the Mathieu equation ${ }^{13}$ suggests that the size is $O\left[\left(\mu e^{2}\right)^{n}\right]$ which is very small. If both $\mu$ and $\lambda$ are nonzero, the lowest order perturbation theory contribution to the gap which wants to open at energy $[(n-k) / n]^{2}$ is, for fixed $k, O\left[\lambda(\mu n)^{k}\right]$, the $n$ 's coming from small energy denominators in perturbation theory. This argument suggests that when $\lambda$ and $\mu$ are small but $\mu n \sim 1$, (2) has, in addition to its "main" gap of size $\lambda$ near energy 1, many other "side gaps."

Now consider a test particle moving in a Keplerian orbit, $v_{0}(t)$, in the equatiorial plane of Saturn in an idealized situation where we initially ignore the moons and the sun. As is well known all these orbits are stable. Now study the effect of the moons with small inclinations. ${ }^{14}$ One should apply the linear stability equation to an actual or bit, $v(t)$, near $v_{0}(t)$, but this orbit is obviously very difficult to compute exactly and indeed it may not even exist in situations where linear stability predicts unstable orbits. We therefore make an approximation: Let $\overrightarrow{\mathrm{F}}_{0}(r)$ and $\overrightarrow{\mathrm{F}}_{1}(r)$ denote the forces on a ring particle due to Saturn and the moons, respectively, so $\ddot{\vec{v}}_{0}=\vec{F}_{0}\left(\vec{v}_{0}\right)$. Since $\overrightarrow{\mathrm{F}}_{1}$ is small an approximate solution is $\overrightarrow{\mathrm{v}} \sim \overrightarrow{\mathrm{v}}_{0}+\overrightarrow{\mathrm{u}}$ with

$$
\ddot{\overrightarrow{\mathrm{u}}}=(\overrightarrow{\mathrm{u}} \cdot \nabla) \cdot \overrightarrow{\mathrm{F}}_{0}\left[\overrightarrow{\mathrm{v}}_{0}(t)\right]+\overrightarrow{\mathrm{F}}_{1}\left[\overrightarrow{\mathrm{v}}_{0}(t)\right] .
$$

$\nabla \overrightarrow{\mathrm{F}}_{0}$ has eigenvalues $\left[\left(2 \pi / T_{r}\right)^{2},\left(2 \pi / T_{r}\right)^{2}, 0\right]$ with $T_{r}$ the period of the Keplerian orbit and $\vec{F}_{1}=\sum \vec{q}_{i}$, $\vec{q}_{i}$ the disturbance function

$$
\begin{aligned}
\overrightarrow{\mathrm{q}}_{i}=\nabla\left\{G m _ { i } \left[\left|\overrightarrow{\mathrm{v}}_{0}(t)-\overrightarrow{\mathrm{r}}_{i}(t)\right|^{-1}\right.\right. \\
\left.\left.-\left|r_{i}(t)\right|^{-3} \overrightarrow{\mathrm{v}}_{0}(t) \cdot \overrightarrow{\mathrm{r}}_{i}(t)\right]\right\} .
\end{aligned}
$$

$m_{i}$ are the masses and $\vec{r}_{i}$ the distance from Saturn of the satellite $i$. $\overrightarrow{\mathrm{u}}(t)$ can be calculated by classical perturbation methods. ${ }^{15}$

If we apply linear stability to $v_{0}+\mu$, we find for the virtual perturbation $w$

$$
\begin{aligned}
\ddot{w}=\left(\nabla_{z} F_{0}\right)\left[v_{0}(t)\right] w & +\nabla_{z} F_{1}\left[u_{0}(t)\right] w \\
& +\left(\nabla_{z} u \cdot \nabla F_{0}\right)\left[u_{0}(t)\right] w,
\end{aligned}
$$

where we keep the first variation of the perturbation $F_{1}$ and the first and second variation of $F_{0}$. Since $\nabla_{z} F_{0}=\left(2 \pi / T_{r}\right)^{2}$, the period, and hence the radius, correspond to the energy in a Schrodinger equation. The situation here is somewhat complicated by the fact that the potential $V(t)$ is a (slowly varying) function of the period. In units of $\left(2 \pi / T_{r}\right)^{2}$, the moon $i$ contributes to the $\nabla F_{1}$ term in (5) an amplitude of the order $\left(m_{i} / m_{\Omega}\right) \mid \boldsymbol{r}_{i} /$ $\left.\left(r-r_{i}\right)\right|^{3}$. This is of order $10^{-7}$ for five moons of Saturn. The contribution to the $\left(\nabla \nabla F_{0}\right) u$ term has amplitude of order $\left(m_{i} / m_{\Omega}\right)\left|r_{i} /\left(r-r_{i}\right)\right|^{3}\left\{n^{2}\left[\left(\Omega_{i} /\right.\right.\right.$ $\left.\left.\left.\omega_{r}\right)-1\right]^{2}\right\}^{-1}$, where $n$ is the driving harmonic. This is again typically of order $10^{-7}$ unless $\omega_{r}$ is near resonance and the curly brackets are small. Thus the second term dominates near resonances. A simple perturbation argument for the Hill's equation gives an estimate of the width of an individual gap due to the moon Mimas alone at the Cassini division to be of order of $20 \mathrm{~km}$, which agrees with numerical solutions of the threebody problem. ${ }^{1}$ Consider now the effects of Mimas and the sun together. The sun has a long period of $\sim 10^{4} \mathrm{~d}$. The amplitude in $(\overrightarrow{\mathrm{u}} \cdot \nabla) F$ due to the sun is of order $\Omega_{\odot} / \omega_{r} .{ }^{16}$ This gives a situation like Eq. (2) with $\mu n$ of order unity providing 
a mechanism for producing side bands. This resolves a serious difficulty that the theory of satellite resonance of Mimas alone had, namely, that Mimas' gap is too small, by orders of magnitude, to account for the Cassini division. In the full problem, apparent gaps can be many orders of magnitude larger than the individual gaps.

Estimating the size of individual gaps (grooves) inside the large apparent rings is more delicate (as is the shift of the center of the Cassini division). In fact, low orders of perturbation theory give unphysically small gaps in this region. We would like to believe that this structure is related to the interplay of the various periods so that small demoninators make low orders of perturbation theory suspect. If true, the structure of the rings away from moon resonances requires an understanding of the almost periodic Schrodinger equation beyond what we currently have.

In summary, we feel that a resonance picture can describe some of the observed qualitative features of the rings of Saturn although it remains to be seen whether further study of the almost periodic Hill operator will verify the intuition based on perturbation theory and whether, even if viable, nature choses this mechanism. We emphasize that linear stability is a single particle theory and is difficult to reconcile if there are many collisions. A competing theory to the satellite resonance of Goldreich and Tremaine ${ }^{17}$ posits the gross ring structure as a collective phenomenon.

We thank J. Moser and R. Johnson for telling us of their work before publication, A. Dekel, L. Yaffe, and particularly P. Goldreich for useful discussions and criticism, I. Mikolic-Torreira for writing a computer program for us, and F. Dyson and P. Lax for encouragement. We would also like to thank the S. Fairchild Visiting Scholar Program and the California Institute of Technology Mathematics Department for their hospitality. This research is supported in part by the National Science Foundation under Grant No. MCS-7801885.

\footnotetext{
(a) On leave from Physics Department, Princeton University, Princeton, N. J. 08544.

(b) On leave from Physics and Mathematics Departments, Princeton University, N. J. 08544. Sherman Fairchild Visiting Scholar at California Institute of Technology, 1980-81.
}

${ }^{1}$ J. B. Pollack, Space Sci. Rev. 18 , 3 (1975); F. A. Franklin et al ., Icarus 12, 338 (1970), and 15, 80 (1971).

${ }^{2}$ See the discussion by V. Arnold, in Classical Mechanics (Springer, New York, 1978).

${ }^{3}$ We emphasize that we are not saying that the rings of Saturn have anything to do with quantum mechanics; only that an equation is the same so quantum mechanical intuition is useful.

${ }^{4}$ This is consistant with the number obtained by Franklin et al ., Ref. 1, by numerically integrating the restricted three-body problem.

${ }^{5}$ We examined two other candidates; precession of the quadrupole moment of Saturn due to Titan and precession of this moment due to the sun. The former has too small an amplitude because the angle of precession is exceedingly small (about $10^{-5} \mathrm{rad}$ ) and this angle enters quadratically; the latter has such a large period (about $10^{10} \mathrm{~d}$ ) that it operates on a scale where a single particle theory must be inadequate.

${ }^{6} \mathrm{We}$ emphasize that there is earlier important work on almost periodic Schrodinger operators; in addition to that of J. Moser (to be published), M. Ya Azbel \{Phys. Rev. Lett. 43, 1954 (1979); see also Zh. Eksp. Teor. Fiz. 44, 980 (1963) ISov. Phys. JETP 17, 665 (1963)], and 46, 929 (1964) $[19,634$ (1964)] $\}$, and D. R. Hofstadter [Phys. Rev. B 14, 2239 (1976)]; we mention B. A. Dubrovin et al ., Russian Math. Surveys 31,59 (1976); E. Dynaburg and Ja. G. Sinai, Functional. Anal. Appl. 9 , 8 (1975); S. Aubry, Ann. Israel Phys. Soc. $\underline{3}$, $133(1 \overline{9} 80)$; R. Johnson, to be published; J. Bellisard and D. Testard, to be published.

${ }^{7}$ Because of small divisors, perturbation theory diverges but it should be a useful guide.

${ }^{8} \mathrm{~A}$ typical limit periodic potential is $\Sigma a_{n} \cos \left(x / 2^{n}\right)$ with $\Sigma\left|a_{n}\right|<\infty$.

${ }^{9}$ See Moser, Ref. 6. While our work (J. Avron and B. Simon, to be published) is independent of Moser's, his certainly precedes ours by some months.

${ }^{10}$ See Avron and Simon, Ref. 9.

${ }^{11}$ See Azbel, Ref. 6.

${ }^{12}$ See Hofstadter, Ref. 6.

${ }^{13} \mathrm{E}$. Harrell, to be published; see also J. Avron and

B. Simon, to be published.

${ }^{14}$ Iapetus and Phoebe have large inclinations but their effect is much smaller than the five main moons.

${ }^{15}$ W. M. Smart, Celestial Mechanics (Longmans, Green, New York, 1953); D. Brower and G. M. Clemence, Methods of Celestial Mechanics (Academic, New York, 1961).

${ }^{16}$ Although the disturbance function is of order $\Omega /$ $\left.\omega_{r}\right)^{2}$, it is known from Delauny lunar theory that there are corrections to the orbit of order $\gamma\left(\Omega_{\odot} / \omega_{r}\right)$ with. period $2 \Omega$ where $\gamma=\sin I$, with $I$ the inclination. $\gamma$ $\sim 0.45$ in the present case. Consequently, one has to consider the full matrix valued Hill's operator.

${ }^{17} \mathrm{P}$. Goldreich and S. Tremaine, Icarus $\underline{34}, 227-239$, 240-253 (1978). 